\title{
Grounded Coplanar Waveguide-fed Compact MIMO Antenna for Wireless Portable Applications
}

\author{
Hamed TAGHIZADEH ${ }^{l}$, Changiz GHOBADI ${ }^{2}$, Burhan AZARM ${ }^{2}$, Maryam MAJIDZADEH ${ }^{3}$ \\ ${ }^{1}$ Electrical Engineering Department, Aeen Kamal University, Urmia, Iran \\ ${ }^{2}$ Electrical Engineering Department, Urmia University, Urmia, Iran \\ ${ }^{3}$ Department of Electrical and Computer Engineering, Urmia Girls Faculty, West Azarbaijan branch, Technical and \\ Vocational University (TVU), Urmia, Iran
}

hamed.taghizadeh16@gmail.com,ch.ghobadi@urmia.ac.ir,st_b.azarm@urmia.ac.ir,,mmajidzadeh@tvu.ac.ir

Submitted February 19, 2019 / Accepted July 11, 2019

\begin{abstract}
A multi-input multi-output (MIMO) antenna with high isolation capability is proposed in this paper. The proposed MIMO antenna configuration is composed of two monopole antennas, each of them consists of a single rectangular grounded coplanar waveguide $(G C P W)$ feed line, a radiation patch with two arms, two conductive elements on both sides of the feed line, and a simple ground plane on substrate backside. The overall size of the proposed MIMO antenna is $44 \times 20 \mathrm{~mm}^{2}$ on $1.6 \mathrm{~mm}$ thick FR4 substrate which is more compact than many of the previously designed structures. The arrangement of two monopole antennas in the form of a MIMO antenna topology yields a dual-band operation in which the first bandwidth is in 3.06-3.89 GHz with the central frequency at $3.5 \mathrm{GHz}$ for WiMAX applications, and 5.14-5.93 GHz with central frequency of $5.5 \mathrm{GHz}$ for WLAN applications. Interestingly the obtained isolation level is better than $-20 \mathrm{~dB}$ over the operating bandwidths. Simulation and measured results confirm the antenna outperformance in WiMAX and WLAN frequency range in wireless portable applications. Small size, simple structure, and high isolation without any decoupling elements are some of the advantages of the proposed design.
\end{abstract}

\section{Keywords}

Monopole antennas, multiple-input-multiple-output (MIMO), WiMAX applications, WLAN application, wireless portable devices

\section{Introduction}

Admirable features of MIMO technology such as the ability in channel capacity increment without the need for extra frequency spectrum, has brought this technology at the center of the researchers attention in communication systems research area. MIMO systems are based on the utilization of more than one antenna in both transmitter and receiver side. Such a configuration could be easily handled in cases with large space without any problem about size issue. However, for portable devices with limited space, the installation of MIMO antennas is a challenge. This is mainly due to the fact that the use of multi antennas inevitably makes the MIMO system a large one. Hence, there has been a trend toward miniaturization of MIMO antennas in recent years [1-3]. The isolation of the adjacent antennas is the other topic which has attracted the attention of researchers. The coupling of the antennas deteriorates the performance of the antennas which is undesired in MIMO systems. Many methods are applied in MIMO antennas to remove or at least to minimize this unwanted coupling [4-7]. For instance in [4], a periodic fractal defected ground structure is used to reduce the mutual coupling which enhances the isolation about $20 \mathrm{~dB}$ and increases the antenna efficiency and channel capacity. Simultaneously, to overcome the cross polarization level degradation, an arc-shaped element is etched under each of the radiating patches to suppress the cross-polarization level. In [5], a line slot is etched to cancel the coupling in $3-4 \mathrm{GHz}$. Moreover, a T-shaped slot is adopted on the ground plane which both enhances the impedance matching and isolation between the antenna elements for the frequencies more than $4 \mathrm{GHz}$. As another example, in [6] the authors have reported a MIMO antenna structure in which the isolation is improved by using carbon black film. The suitable utilization of the film yields absorption of the interference between the constituent antenna elements. In [7] the antenna elements are closely placed in the form a MIMO antenna, but the high isolation is achieved by adopting two decoupling methods, a meandering resonant branch and an inverted T-shaped slot etched on the ground for the higher band and the lower band, respectively.

Although many problems are faced in design process of MIMO antennas, their popularity is increasing every day as many interesting applications of them are revealed. Among them, Long Term Evolution (LTE) applications [8-10], ultra wide band applications [11], [12], C and X band applications [13], and WiMAX applications [14] could be named. 
This paper aims at designing and discussing the performance of a novel MIMO antenna with compact size, high isolation, and special application in wireless portable devices. The proposed two-element MIMO antenna has a compact size of $44 \times 20 \mathrm{~mm}^{2}$ which is printed on $1.6 \mathrm{~mm}$ thick FR4 substrate. Grounded coplanar waveguide (GCPW) feed line excites the antenna. The conductive elements are so designed to focus the resonances at $3.5 \mathrm{GHz}$ and $5.5 \mathrm{GHz}$ and generate a dual-band performance at WiMAX and WLAN frequency bands. More interestingly the obtained isolation level is better than $-20 \mathrm{~dB}$ which is obtained without any extra element between the antennas. Hence, in comparison with other MIMO antenna structures, the proposed antenna has the advantage of being simpler and more cost effective due to the absence of any decoupling conductive element between the antennas. This is while in most of the other MIMO antennas, some kind of decoupling element is adopted. The main contributions of this paper are summarized as follows:

- Proposing a novel scheme of a single microstrip antenna with dual-band performance.

- Developing a two-element MIMO antenna with tuned operating bandwidths at WiMAX and WLAN applications.

- Obtaining a high value of isolation between antennas without any extra parasitic elements between the antennas.

Moreover, compared with previously designed structures, the proposed antenna is smaller which makes it a cost effective and compact one. Also, the high level of isolation is obtained without any decoupling mechanism adoption which is mainly due to the perpendicular alignment of the antennas with respect to each other.

In the following, Section 2 presents the single microstrip antenna design and performance analysis. Then, in Sec. 3 MIMO antenna topology as well as the structural and technical performance features are discussed in detail. In the sequel, the advantages of the proposed MIMO antenna are highlighted over the similar previous designs in Sec. 4. Finally, Section 5 concludes the paper.

\section{Single Monopole Antenna Design and Performance}

The monopole antenna configuration is depicted in Fig. 1. As it was mentioned before, the antenna is composed of a simple rectangular ground plane on the substrate backside. As well, on top side, a GCPW feeds the radiation patch which is composed of two arms. Two rectangular conductive elements are also embedded on both sides of the GCPW. It is worth noting that in GCPW structures, there is an extra ground plane on the substrate backside which helps to enhance the antenna performance over the operating frequency bands. Each of the constituent arms of the radiating patch is responsible for one of the obtained operating frequency bands. In fact, different resonances are excited by these arms which result in a dual-band operation. It will be shown that by tuning the dimensions of these arms, the central resonance frequencies could be effectively tuned at the desired frequency. FR4 substrate with thickness of $1.6 \mathrm{~mm}$, relative dielectric constant of 4.4 , and loss tangent of 0.02 is adopted as the antenna substrate. The optimized values of the antenna parameters are reported in detail as follows based on the obtained value from HFSS. All the unit values are in millimeter $(\mathrm{mm}): L_{\text {sub }}=20 \mathrm{~mm}, W_{\text {sub }}=22 \mathrm{~mm}, W_{\mathrm{g}}=20 \mathrm{~mm}, L_{\mathrm{g}}=$ $4 \mathrm{~mm}, W_{\mathrm{f}}=2 \mathrm{~mm}, L_{\mathrm{f}}=11 \mathrm{~mm}, W_{\mathrm{f} 1}=4 \mathrm{~mm}, L_{\mathrm{f} 1}=7 \mathrm{~mm}$, $W_{\mathrm{x}}=4.5 \mathrm{~mm}, \quad L_{\mathrm{y}}=0.5 \mathrm{~mm}, \quad g=0.6 \mathrm{~mm}, \quad d x=2.5 \mathrm{~mm}$, $L_{\mathrm{x} 1}=5 \mathrm{~mm}, L_{\mathrm{y} 1}=3.5 \mathrm{~mm}, L_{\mathrm{y} 2}=1.5 \mathrm{~mm}, L_{\mathrm{x} 2}=3.5 \mathrm{~mm}$, $W_{\mathrm{x} 1}=W_{\mathrm{x} 2}=2 \mathrm{~mm}$,

To better understand the antenna design process regarding the dual band functionality and reveal the technical performance, the proposed monopole antenna is developed in a two-stage process as shown in Fig. 2. As can be seen, in stage 1 , only $1 \mathrm{arm}$ is connected to the feed line as the radiation patch. In stage 2 , the second arm is also connected to finalize the antenna structure. The ground plane, two rectangular elements, and the feed line are similar in both designs. Ansoft High Frequency Structural Simulator (HFSS) software is used to simulate both antenna structures

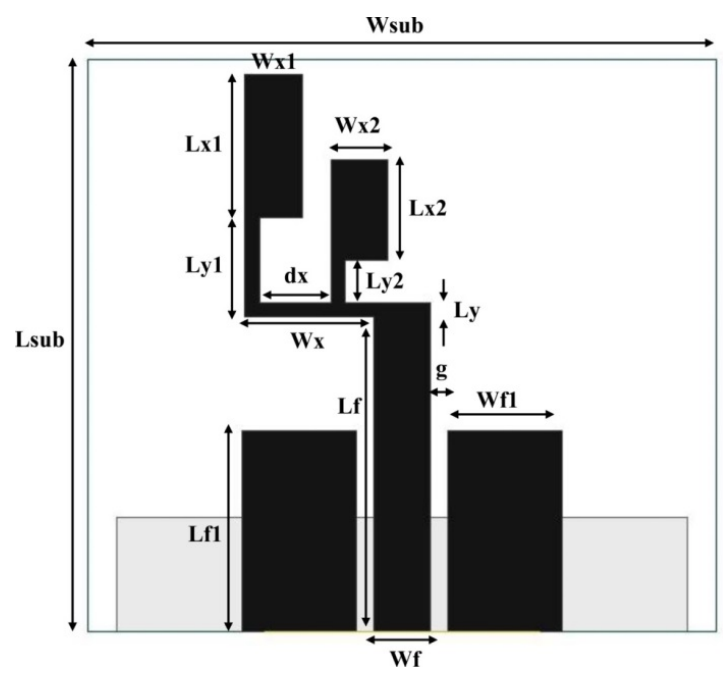

Fig. 1. Geometry of the proposed single monopole antenna.

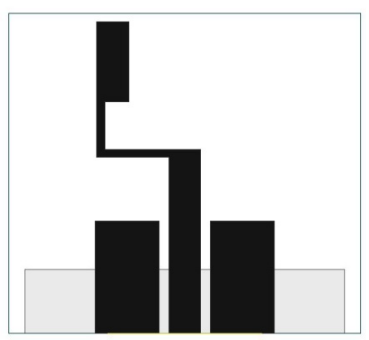

(a)

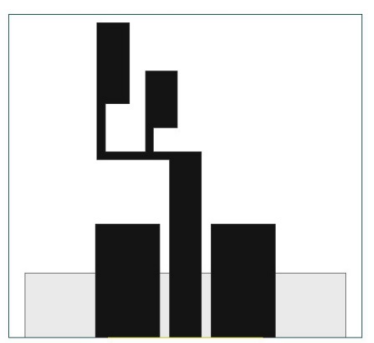

(b)
Fig. 2. Design process of the proposed dual band monopole antenna: (a) Antenna with one arm in the radiating patch. (b) Antenna with two arms in the radiating patch. 


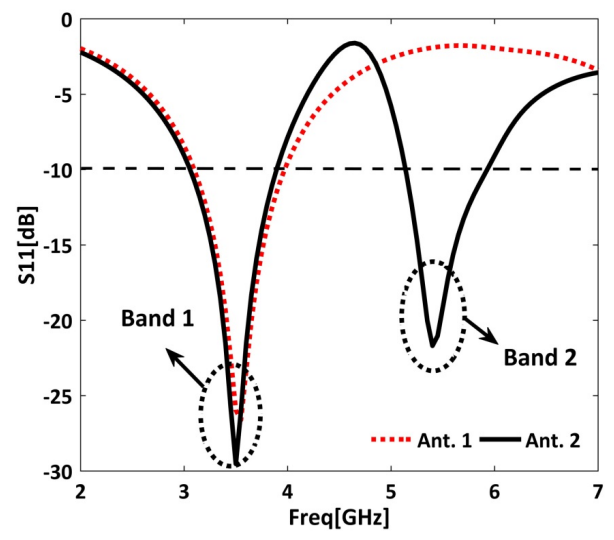

Fig. 3. $S_{11}$ curves for the antennas in Fig. 2.

and the resultant $S_{11}$ curves are plotted in Fig. 3. As it is clearly seen, in stage 1 , when only one arm is embedded in the antenna body, one resonance is excited at $3.5 \mathrm{GHz}$. In this case the frequency band of $3.06-3.89 \mathrm{GHz}$ is covered by the antenna. This is while, by the inclusion of the other arm, another resonance is also seen in $5.5 \mathrm{GHz}$ which generates the second operating bandwidth from 5.14 to $5.93 \mathrm{GHz}$. Interestingly the inclusion of the second arm does not affect the resonance frequency at $3.5 \mathrm{GHz}$.

Surface current distribution is analyzed to reveal the role of each arm of the radiation patch on the antenna performance. Figure 4(a) shows the surface current distribution at $3.5 \mathrm{GHz}$ which is the central frequency of the first operating bandwidth. As it was expected from the abovementioned discussion, the first arm contains the dominant radiating current which confirms the effect of this arm on the excitation of the resonance at $3.5 \mathrm{GHz}$. Moreover, Figure 4(b) clearly shows that at $5.5 \mathrm{GHz}$, the current is mostly concentrated on the lower parts of the second arm. This clearly means that this arm is directly responsible for the excitation of the second resonance and consequently the second operating bandwidth generation. As can be seen, this observation is in line with the conclusions made in antenna design process regarding the role of each constituent element.

Simulated gain of the proposed single antenna is plotted in Fig. 5. It is seen that a gain value of about $4 \mathrm{dBi}$ is obtained over both operating bandwidths which is considered as a suitable one.

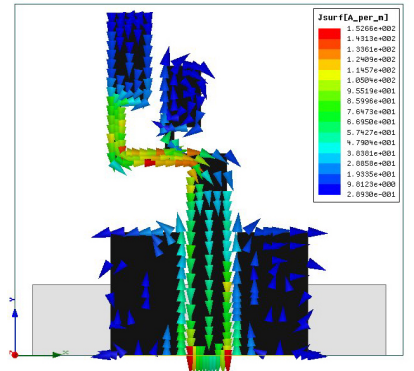

(a)

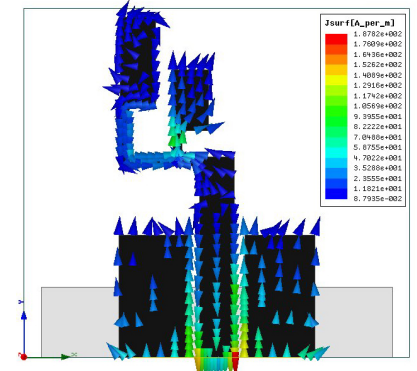

(b)
Fig. 4. Surface current distribution on the proposed single antenna (a) $3.5 \mathrm{GHz}$, (b) $5.5 \mathrm{GHz}$.

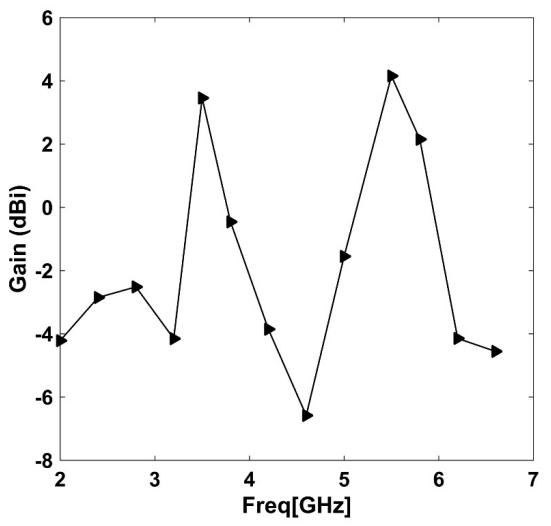

Fig. 5. Gain curve for the proposed single antenna.

\section{MIMO Antenna Design and Analysis}

The proposed MIMO antenna topology is shown in Fig. 6. Two monopole antennas are suitably placed perpendicular to each other to construct a MIMO system. It should be noted that the perpendicular placement of the antennas usually yields a high isolation between the antennas and as a consequence there will be no need for decoupling structure between the antennas. This, in turn reduces the cost and complexity of the MIMO antenna topology. The overall size of the MIMO antenna is $44 \times 20 \mathrm{~mm}^{2}$ which is much more compact with respect to many of the similar previously designed MIMO antennas. $S_{11}$ and $S_{21}$ curves for the proposed MIMO antenna are shown in Fig. 7. Similar to the single monopole antenna, the MIMO antenna exhibits a dual-band operation focused at $3.5 \mathrm{GHz}$ and $5.5 \mathrm{GHz}$ frequencies. Moreover, the isolation level is better than $-20 \mathrm{~dB}$ over the entire frequency band. Interestingly this value of isolation is obtained without any contrivance regarding the coupling effect decrement.

One of the important features in MIMO antenna design is the envelope correlation coefficient (ECC). In fact ECC shows how independent the constituent antennas act. The lower is the ECC, the more efficient is the antenna. ECC is calculated based on (1) [17]

$$
E C C=\frac{\left|S_{11}{ }^{*} S_{12}+S_{21}{ }^{*} S_{22}\right|^{2}}{\left(1-\left|S_{11}\right|^{2}-\left|S_{21}\right|^{2}\right)\left(1-\left|S_{22}\right|^{2}-\left|S_{12}\right|^{2}\right)} .
$$

Figure 8 shows the ECC curve for the proposed MIMO antenna. As can be seen, less than 0.02 ECC is obtained over the operating bandwidth.

To validate the obtained results of the proposed MIMO antenna, a prototype is fabricated for measurement purpose. Figure 9 shows the fabricated antenna in test process. As can be seen, the antenna is connected to PNA (EVA368) E8363C for $\mathrm{S}$ parameters measurement. The obtained $S_{11}$ and $S_{21}$ curves are plotted in Fig. 10, and are compared with the simulated ones. As can be seen in this figure, the results are in good agreement with each other. 


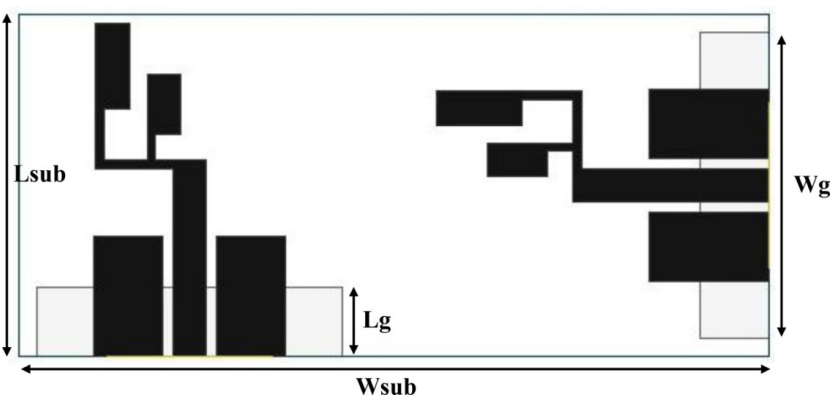

Fig. 6. The proposed dual band MIMO antenna configuration.

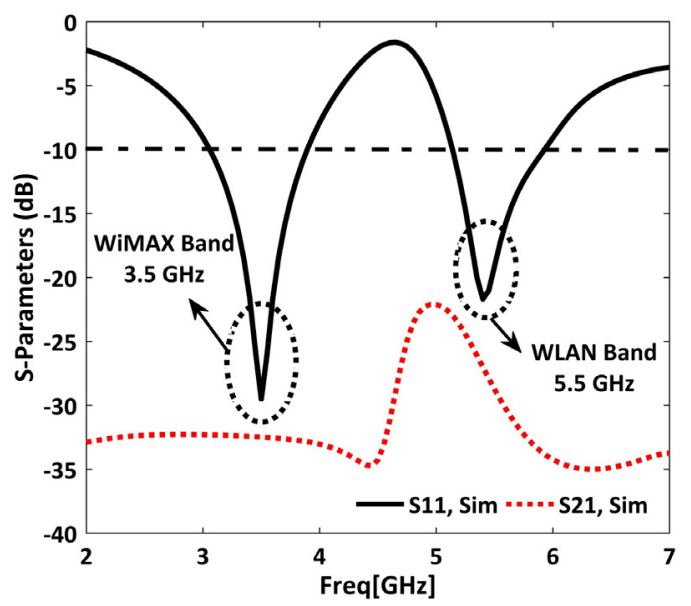

Fig. 7. $S_{11}$ and $S_{21}$ curves for the proposed MIMO antenna configuration.

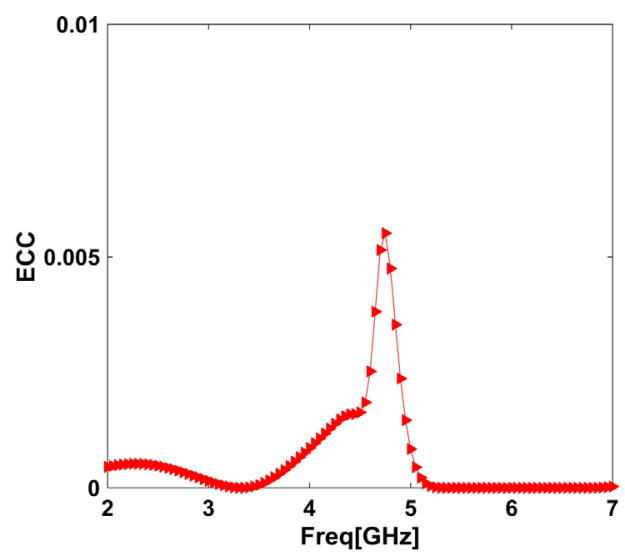

Fig. 8. Simulated ECC for the proposed MIMO antenna configuration.

The obtained results confirm the antenna performance in $3.06-3.89 \mathrm{GHz}$ for WiMAX, and in $5.14-5.93 \mathrm{GHz}$ for WLAN frequency range. In both operating bands, better than $-20 \mathrm{~dB}$ isolation is achieved.

Moreover, the antenna is put under radiation pattern measurement in antenna anechoic chamber, too. Figure 11 shows the fabricated MIMO antenna in radiation pattern measurement process. Simulated and measured E-plane and H-plane patterns are shown in Fig. 12 at $3.5 \mathrm{GHz}$ and $5.5 \mathrm{GHz}$ which are the central frequencies of the operating bandwidths. It is worth noting than in measurement process, one port is excited and the other port is connected to a standard $50 \Omega$ load. As the results indicate, omnidirec-
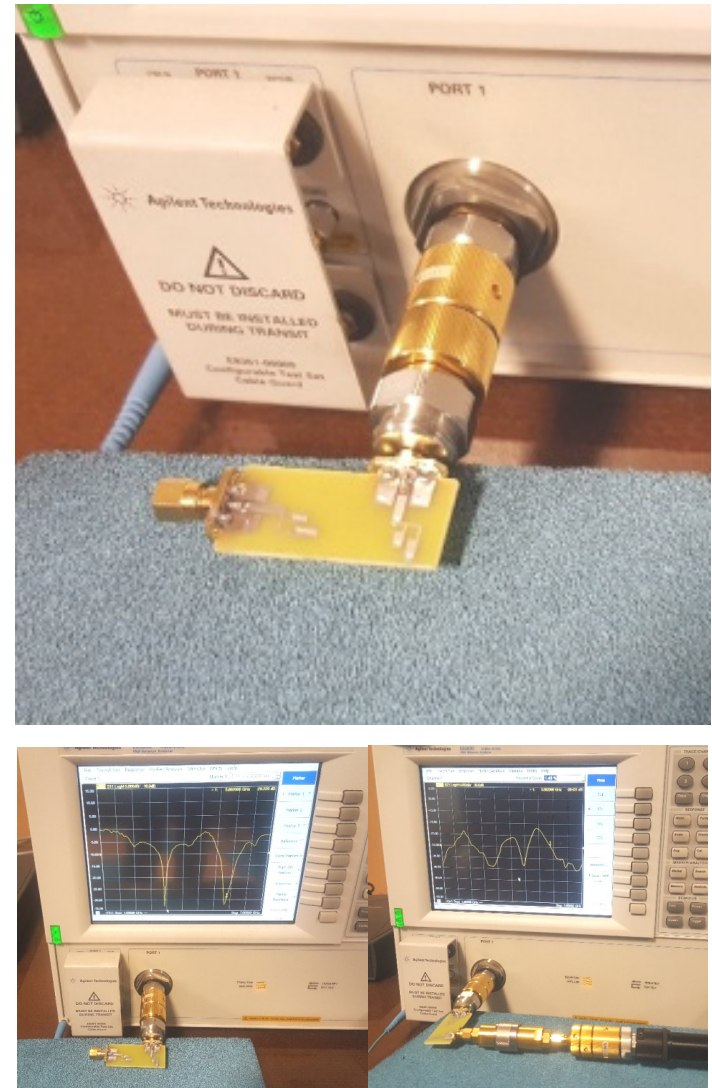

Fig. 9. The fabricated MIMO antenna in S-parameters measurement process.

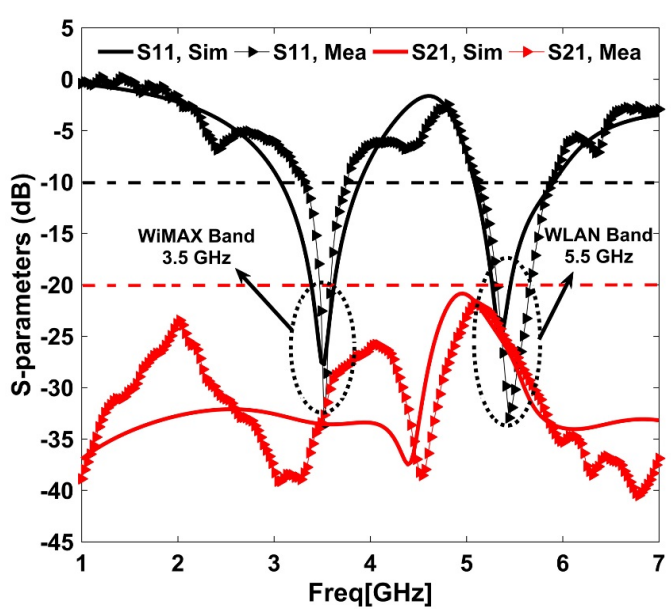

Fig. 10. Simulated and measured $S_{11}$ and $S_{21}$ curves.

tional radiation pattern is seen at both frequencies which shows the antenna suitable performance. As well, the agreement of the simulated and measured results indicates the reliable performance of the antenna in real-world applications. The small discrepancies could be due to the fabrication errors, the human being and also the measurement setup errors, which could be neglected.

Simulated and measured peak gain and radiation efficiency of the proposed MIMO antenna are plotted in Fig. 13. A peak gain of about $4 \mathrm{dBi}$ as well as an efficiency of about $90 \%$ is obtained in both operating bands. Moreover, the antenna group delay is shown in Fig. 14. The result 
confirms less than 1 nanoseconds (ns) variation for the antenna which lays in the standard acceptable range for the MIMO systems.

In addition, the simulated and measured ECC is shown in Fig. 15. Based on the explanations provided in

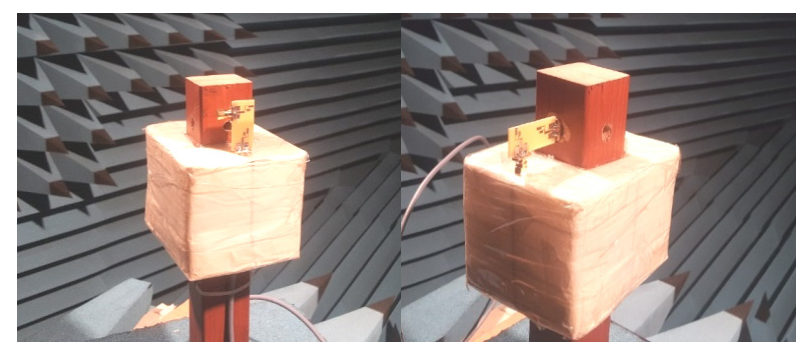

Fig. 11. The proposed MIMO antenna in radiation pattern measurement process.

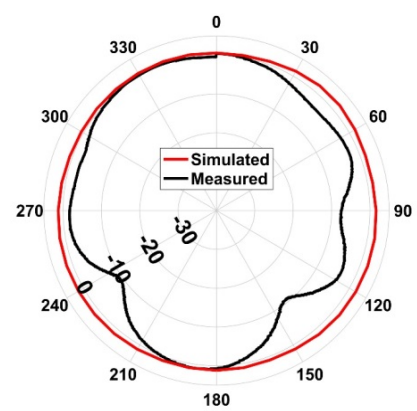

(a)

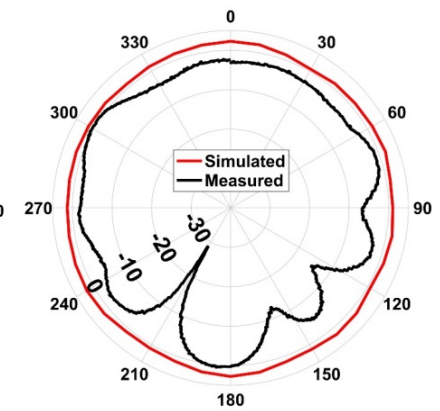

(b)

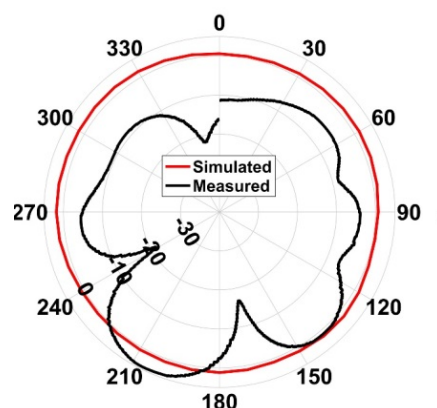

(c)

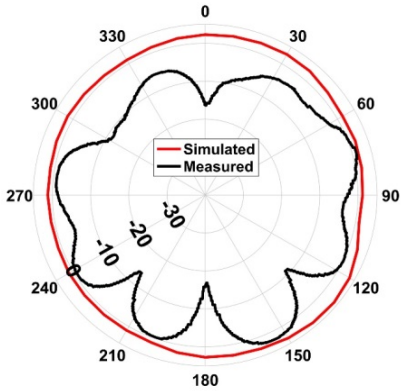

(d)
Fig. 12. The proposed MIMO antenna radiation patterns: (a) $3.5 \mathrm{GHz}$ (y-z plane), (b) $5.5 \mathrm{GHz}$ (y-z plane), (c) $3.5 \mathrm{GHz}$ (x-z plane, and (d) $5.5 \mathrm{GHz}$ (x-z plane).

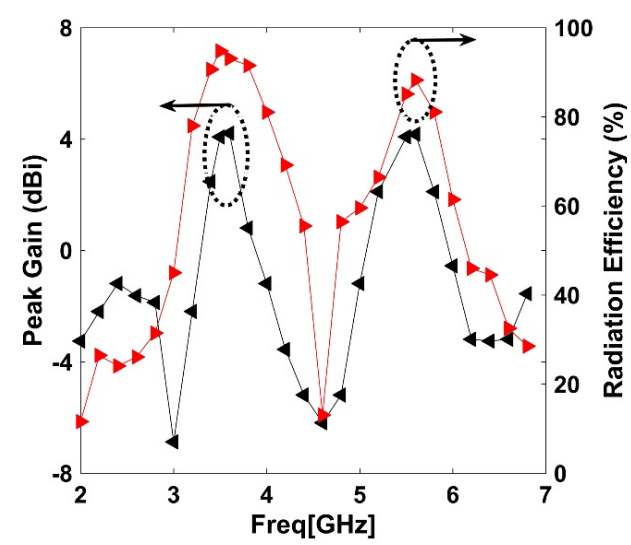

Fig. 13. Simulated and measured peak gain and radiation efficiency of the proposed MIMO antenna. previous sections, the obtained ECC values fall within the acceptable range for communication applications.

The effect of adjacency of antennas should be analyzed in detail in a MIMO system. To this end, apart from ECC, another parameter is also studied. This important metric is named as total active reflection coefficient (TARC) which is defined as the square root of the ratio of total reflected power to the total incident power and it's apparent return loss of the overall MIMO antenna system.

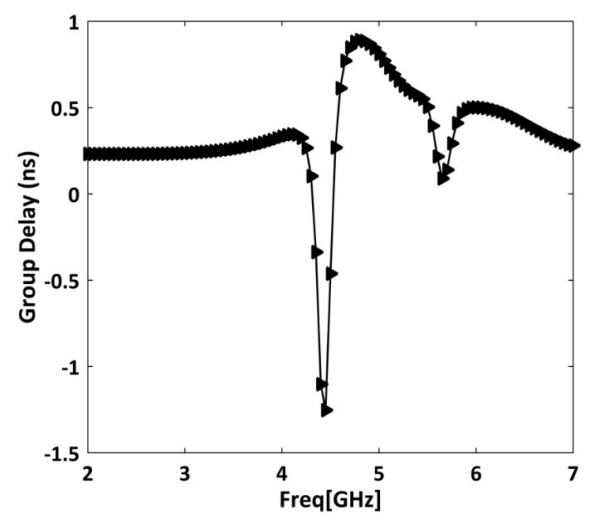

Fig. 14. Simulated group delay of the proposed MIMO antenna.

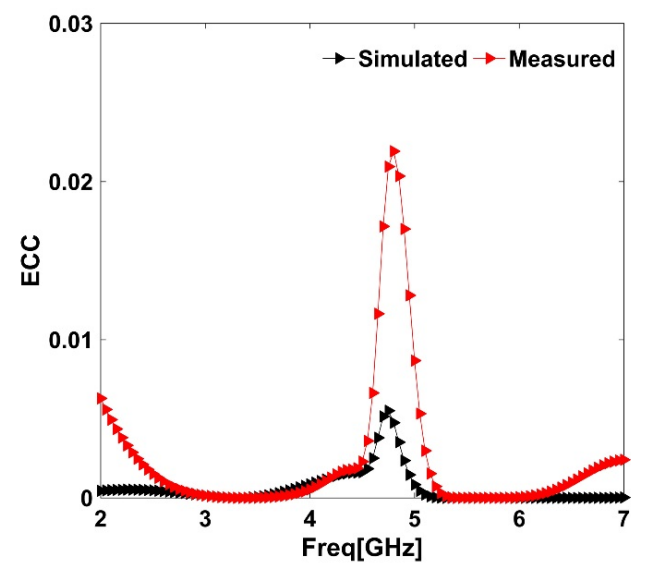

Fig. 15. Simulated and measured ECC of the proposed MIMO antenna.

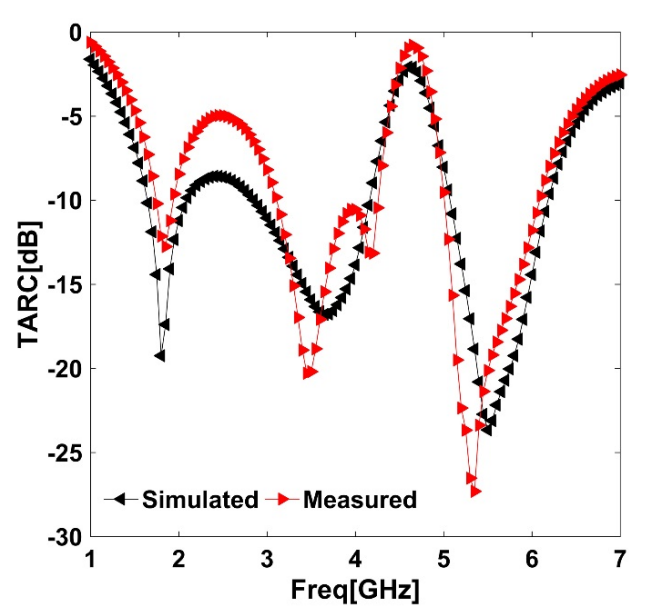

Fig. 16. Simulated and measured TARC of the proposed MIMO antenna. 
For two-element MIMO antenna, TARC is calculated as follows [17]:

$$
T A R C=\sqrt{\frac{\left(S_{11}+S_{12}\right)^{2}+\left(S_{21}+S_{22}\right)^{2}}{2}} .
$$

Less than $0 \mathrm{~dB}$ values are desired in MIMO systems. As Fig. 16 indicates, this requirement is fully met in the proposed MIMO antenna system.

\section{Comparison with Previously Published Similar Configurations}

To highlight the superiority of the proposed MIMO antenna over some of the previous similar designs, a comparison is carried out in Tab. 1. The comparison terms include the number of elements in MIMO antennas, central frequency of the operating bandwidths, size, and isolation. The summarized data in Tab. 1 reveal that the antenna in [13] is the smallest between the antennas in comparison but low isolation level is obtained. This is while the antenna in [15] with four constituent antennas offers two operating bands with $-17.5 \mathrm{~dB}$ isolation. Also, the proposed antenna in [16] is larger than the antenna in this work and operates in three frequency bands with the same isolation as the antenna in this work. Specifically speaking, the proposed antenna in this work has provided a suitable trade-off between all the important structural and technical features.

\section{Conclusion}

A dual-band GCPW-fed MIMO antenna with high isolation is proposed for portable wireless systems. A simple radiation patch with two conductive elements and a simple rectangular ground plane is the structure of each constituent single antenna. Arranging two single antennas in a MIMO form, yields the coverage of two operating frequency bands with central frequencies of $3.5 \mathrm{GHz}$ and 5.5 GHz for WiMAX and WLAN applications. As an interesting technical feature, no parasitic element is adopted to increase the isolation, but the arrangement of the two antennas itself provides the high level inter-element isolation. Small size, wide bandwidth, and simple structure are some of the advantages observed by the proposed design. Moreover, close agreement was achieved between the simulated and measured results which confirms that the proposed MIMO antenna is a suitable choice for portable

\begin{tabular}{|c|c|c|c|c|}
\hline Ref & $\begin{array}{c}\text { Element } \\
\text { number }\end{array}$ & $\begin{array}{c}\text { Central frequency of } \\
\text { operating bands }\end{array}$ & $\begin{array}{c}\text { Antenna } \\
\text { size }\left(\mathrm{mm}^{2}\right)\end{array}$ & $S_{21}(\mathrm{~dB})$ \\
\hline$[13]$ & 2 & $7.2 / 8.57$ & $17 \times 42$ & -13 \\
\hline$[15]$ & 4 & $2.4 / 5.5$ & $50 \times 50$ & -17.5 \\
\hline$[16]$ & 2 & $2.4 / 5.2 / 5.8$ & $50 \times 26$ & -20 \\
\hline $\begin{array}{c}\text { This } \\
\text { work }\end{array}$ & 2 & $3.5 / 5.5$ & $44 \times 20$ & -20 \\
\hline
\end{tabular}

Tab. 1. Summary of the characteristics of the proposed antennas and some previously designed structures.
UWB MIMO applications. The comparison revealed that the proposed antenna is smaller than many of the previous antennas with more simplicity. Moreover, no decoupling element was adopted to enhance the isolation which is the direct result of suitable alignment of the antennas with respect to each other.

\section{References}

[1] WEN, D., HAO, Y., MUNOZ, M. O., et al. A compact and lowprofile MIMO antenna using a miniature circular high-impedance surface for wearable applications. IEEE Transactions on Antennas and Propagation, 2018, vol. 66, no. 1, p. 96-104. DOI: 10.1109/TAP.2017.2773465

[2] LEE, J., HONG, Y.K., BAE, S., et al. Miniature Long-Term Evolution (LTE) MIMO ferrite antenna. IEEE Antennas and Wireless Propagation Letters, 2011, vol. 10, p. 603-606. DOI: 10.1109/LAWP.2011.2159468

[3] AZARM, B., NOURINIA, J., GHOBADI, C., et al. A compact WiMAX band-notched UWB MIMO antenna with high isolation. Radioengineering, 2018, vol. 27, no. 4, p. 983-989. DOI: $10.13164 /$ re.2018.0983

[4] ZHANG, Z. L., WEI, K., XIE, J., et al. The MIMO antenna array with mutual coupling reduction and cross-polarization suppression by defected ground structures. Radioengineering, 2018, vol. 27, no. 4, p. 969-975. DOI: 10.13164/re.2018.0969

[5] LUO, C. M., HONG, J. S., ZHONG, L. L. Isolation enhancement of a very compact UWB-MIMO slot antenna with two defected ground structures. IEEE Antennas and Wireless Propagation Letters, 2015, vol. 14, p. 1766-1769. DOI: 10.1109/LAWP.2015.2423318

[6] LIN, G. S., SUNG, C. H., CHEN, J. L., et al. Isolation improvement in UWB MIMO antenna system using carbon black film. IEEE Antennas and Wireless Propagation Letters, 2016, vol. 16, p. 222-225. DOI: 10.1109/LAWP.2016.2570301

[7] DENG, J. Y., LI, J. Y., ZHAO, L., GUO, L. A dual-band invertedF MIMO antenna with enhanced isolation for WLAN applications. IEEE Antennas and Wireless Propagation Letters, 2017, vol. 16, p. 2270-2273. DOI: 10.1109/LAWP.2017.2713986

[8] BARANI, I. R. R., WONG, K. L. Integrated inverted-F and openslot antennas in the metal-framed smartphone for $2 \times 2 \mathrm{LTE} \mathrm{LB}$ and $4 \times 4$ LTE M/HB MIMO operations. IEEE Transactions on Antennas and Propagation, 2018, vol. 66, no. 10, p. 5004-5012. DOI: 10.1109/TAP.2018.2854191

[9] DIOUM, I., DIALlO, A., FARSSI, S. M., LUXEY, C. A novel compact dual-band LTE antenna-system for MIMO operation. IEEE Transactions on Antennas and Propagation, 2014, vol. 62, no. 4, p. 2291-2296. DOI: 10.1109/TAP.2014.2301151

[10] KWON, O. Y., SONG, R., KIM, B. S. A fully integrated shark-fin antenna for MIMO-LTE, GPS, WLAN, and WAVE applications. IEEE Antennas and Wireless Propagation Letters, 2018, vol. 17, p. 600-603. DOI: 10.1109/LAWP.2018.2805681

[11] SRIVASTAVA, G., MOHAN, A. Compact MIMO slot antenna for UWB applications. IEEE Antennas and Wireless Propagation Letters, 2015, vol. 15, p. 1057-1060. DOI: 10.1109/LAWP.2015.2491968

[12] REN, J., HU, W., YIN, Y., FAN, R. Compact printed MIMO antenna for UWB applications. IEEE Antennas and Wireless Propagation Letters, 2014, vol. 13, p. 1517-1520. DOI: 10.1109/LAWP.2014.2343454 
[13] POUYANFAR, N., GHOBADI, CH., NOURINIA, J., et al. A compact multi-band MIMO antenna with high isolation for $\mathrm{C}$ and $\mathrm{X}$ bands using defected ground structure. Radioengineering, 2018, vol. 27, no. p. 686-693. DOI: 10.13164/re.2018.0686

[14] QUDDUS, A., SALEEM, R., SHAFIQUE, M. F., et al. Compact electronically reconfigurable WiMAX band-notched ultrawideband MIMO antenna. Radioengineering, 2018, vol. 27, no. 4, p. $998-1005$. DOI: $10.13164 /$ re. 2018.0998

[15] LIAO, W. J., HSIEH, C. Y., DAI, B. Y., et al. Inverted-F/slot integrated dual-band four-antenna system for WLAN access points. IEEE Antennas and Wireless Propagation Letters, 2015, vol. 14, p. 847-850. DOI: 10.1109/LAWP.2014.2381362

[16] LUO, C. M., HONG, J. S., AMIN, M. Mutual coupling reduction for dual-band MIMO antenna with simple structure. Radioengineering, 2017, vol. 26, no. 1, p. 51-56. DOI: $10.13164 /$ re.2017.0051

[17] CHANDEL, R., KUMAR GAUTAM, A., RAMBABU, K. Tapered fed compact UWB MIMO-diversity antenna with a dual band-notched characteristics. IEEE Transactions on Antennas and Propagation, 2018, vol. 66, no. 4, p. 1677-1684. DOI: 10.1109/TAP.2018.2803134

\section{About the Authors ...}

Hamed TAGHIZADEH was born on 1994 in Iran. He received the B.Sc. degree in Electrical Engineering from the University College of Science and Technology, Urmia, Iran. He is currently working toward the M.Sc degree in $\mathrm{RF}$ and Microwave Engineering at Aeen Kamal University, Urmia, Iran.

Changiz GHOBADI received his B.Sc. in Electrical Engineering-Electronics and M.Sc. degrees in Electrical Engi-
neering-Telecommunication from Isfahan University of Technology, Isfahan, Iran and Ph.D. degree in Electrical Engineering-Telecommunication from University of Bath, Bath, UK in 1998. He is currently a professor in the Department of Electrical Engineering of Urmia University, Urmia, Iran. He established Northwest Antenna and Microwave Research Laboratory (NAMRL) at the University of Urmia with the focus on microwave, antennas and propagation devices characterization, design and fabrication. He has been included in the Top One Percent of the World's Scientists and Academics according to Thomson Reuters' list in 2017. His primary research interests are in antenna design, radar and adaptive filters.

Burhan AZARM was born on 1992 in Iran. He received the B.Sc. degree in Power Engineering from Islamic Azad University, Urmia, Iran. He is currently working toward the M.Sc degree in RF and microwave engineering at Urmia University. His research interests include antennas, microwave, and electromagnetics.

Maryam MAJIDZADEH was born in 1987 in Urmia, Iran. She received her B.Sc. in Electrical Engineering-Electronics from Urmia University in 2009. Then, she received her M.Sc. and Ph.D. degrees in Electrical Engineering-Communication from the same university in 2012 and 2016, respectively. She is now an Assistant Professor in the Department of Electrical and Computer Engineering, Urmia Girls Faculty, West Azarbaijan branch, Technical and Vocational University (TVU), Urmia, Iran. Her research interests include antenna design, antenna miniaturization techniques, frequency selective surfaces, electromagnetic compatibility, MIMO antennas, and filters. 